\title{
A Simple Method for the Preparation of Crude Gintonin from Ginseng Root, Stem, and Leaf
}

\author{
Mi Kyung Pyo ${ }^{1 \#}$, Sun-Hye Choi ${ }^{2 \#}$, Tae-Joon Shin ${ }^{2 \#}$, Sung Hee Hwang ${ }^{2}$, Byung-Hwan Lee ${ }^{2}$, Jiyeon \\ Kang $^{2}$, Hyeon-Joong Kim², Soo-Han Lee ${ }^{3}$, and Seung-Yeol $\mathrm{Nah}^{2 *}$ \\ ${ }^{1}$ International Ginseng and Herb Research Institute, Guemsan 312-804, Korea \\ ${ }^{2}$ Ginsentology Research Laboratory and Department of Physiology, College of Veterinary Medicine and Bio/Molecular \\ Informatics Center, Konkuk University, Seoul 143-701, Korea \\ ${ }^{3}$ Department of Clinical Pharmacology and Therapeutics, University of Ulsan College of Medicine, Seoul 138-736, Korea
}

Ginseng has been used as a general tonic agent to invigorate the human body as an adaptogenic agent. In a previous report, we have shown that ginseng contains a novel glycolipoprotein called gintonin. The main function of gintonin is to transiently enhance intracellular free $\mathrm{Ca}^{2+}\left[\mathrm{Ca}^{2+}\right]_{\mathrm{i}}$ levels in animal cells. The previous method for gintonin isolation included multiple steps using organic solvents. In the present report, we developed a simple method for the preparation of crude gintonin from ginseng root as well as stem and leaf, which produced a higher yield of gintonin than the previous one. The yield of gintonin was $0.20 \%, 0.29 \%$, and $0.81 \%$ from ginseng root, stem, and leaf, respectively. The apparent molecular weight of gintonin isolated from stem and leaf through sodium dodecyl sulfate polyacrylamide gel electrophoresis was almost same as that from root but the compositions of amino acids, carbohydrates or lipids differed slightly between them. We also examined the effects of crude gintonin from ginseng root, stem, and leaf on endogenous $\mathrm{Ca}^{2+}$-activated $\mathrm{Cl}^{-}$channel $(\mathrm{CaCC})$ activity of Xenopus oocytes through mobilization of $\left[\mathrm{Ca}^{2+}\right]$ i. We found that the order of potency for the activation of $\mathrm{CaCC}$ was ginseng root $>$ stem $>$ leaf. The $\mathrm{ED}_{50}$ was $1.4 \pm 1.4,4.5 \pm 5.9$, and $3.9 \pm 1.1 \mu \mathrm{g} / \mathrm{mL}$ for root, stem and leaf, respectively. In the present study, we demonstrated for the first time that in addition to ginseng root, ginseng stem and leaf also contain gintonin. Gintonin can be prepared from a simple method with higher yield of gintonin from ginseng root, stem, and leaf. Finally, these results demonstrate the possibility that ginseng stem and leaf could also be utilized for ginstonin preparation after a simple procedure, rather than being discarded.

Keywords: Panax ginseng, Ginseng, Root, Stem, Leaf, Gintonin

\section{INTRODUCTION}

Ginseng, the root of Panax ginseng, has been used as a general tonic or an adaptogen to promote longevity and enhance bodily functions against stress, fatigue, and various diseases including cancer and diabetes mellitus [1]. Ginseng is one of the most widely used and precious herbal medicines consumed around the world, containing various biologically active ingredients such as ginsenosides (also called ginseng saponins), acidic polysaccharides, polyacetylenes, and other minor components [1]. Among them, ginsenosides were regarded as (cc) This is an Open Access article distributed under the terms of the Creative Commons Attribution Non-Commercial License (http://creativecommons.org/licenses/by-nc/3.0/) which permits unrestricted non-commercial use, distribution, and reproduction in any medium, provided the original work is properly cited.
Received 04 Jan. 2011, Revised 03 May 2011, Accepted 03 May 2011

\#Authors contributed equally to this work.

*Corresponding author

E-mail: synah@konkuk.ac.kr

Tel: + 82-2-450-4154, Fax: +82-2-450-3037 
representative agents of ginseng for pharmacological and physiological investigations. In previous reports, we have demonstrated that crude ginseng total saponin fraction activates endogenous $\mathrm{Ca}^{2+}$-activated $\mathrm{Cl}^{-}$channel $(\mathrm{CaCC})$ in Xenopus oocytes, of which $\mathrm{CaCC}$ channel is activated by transiently mobilizing intracellular $\mathrm{Ca}^{2+}\left[\mathrm{Ca}^{2+}\right]_{\mathrm{i}}[2-4]$. We demonstrated that active agents which caused $\mathrm{CaCC}$ activation in Xenopus oocytes and transiently elevated $\left[\mathrm{Ca}^{2+}\right]_{\mathrm{i}}$ in mammalian cell are not ginsenosides, but rather novel glycolipoproteins called gintonin [5]. Thus, we provided evidence that ginseng contains the novel ingredient gintonin that could also be the primary agent for various $\mathrm{Ca}^{2+}$-mediated cellular events.

The previous methods for crude gintonin preparation included multiple steps using various organic solvents with a time intensive separation process [5]. In present study, we developed a simple method for crude gintonin preparation from ginseng root as well as from stem and leaf with CaCC activation. We report here that this procedure simplified the crude gintonin preparation process and produced a much higher yield of gintonin as compared to the previous method. In addition, the apparent molecular weight of gintonin isolated from stem and leaf was almost same as that of root. Finally, the present report suggests the possibility that ginseng stem and leaf could also be utilized for gintonin preparation, which is usually otherwise discarded.

\section{MATERIALS AND METHODS}

\section{Materials}

BioLogic DuoFlow ${ }^{\mathrm{TM}}$ chromatography systems were purchased from Bio-Rad (Hercules, CA, USA). Six-yearold Korea red ginseng (Korea Ginseng Corporation, Daejeon, Korea) was purchased from a local ginseng market. Prepacked columns (Superdex 75 10/30/300 GL and HiTrap $^{\text {TM }}$ DEAE FF) and media (DEAE Sepharose CL-6B) were purchased from GE Healthcare (Uppsala, Sweden). Schiff's reagent, tubing cellulose membrane with molecular weight cut off at 13,000 and other reagents were all purchased from Sigma-Aldrich (St. Louis, MO, USA).

\section{Preparation of gintonin fraction from ginseng root}

Eight kilograms of 4-year-old ginseng roots were ground into small pieces $(>3 \mathrm{~mm})$ and refluxed with $80 \%$ methanol $(\mathrm{MeOH})$ three times for $8 \mathrm{~h}$ at $80^{\circ} \mathrm{C}$ each. The $\mathrm{MeOH}$ extracts $(1.3 \mathrm{~kg})$ concentrated in vacuo were partitioned between $n$-butanol $(n-\mathrm{BuOH})$ and water. $n$ $\mathrm{BuOH}$ fraction (fr., $300 \mathrm{~g}$ ) of Panax ginseng dissolved in phosphate buffer saline (PBS, pH 7.2) was loaded onto

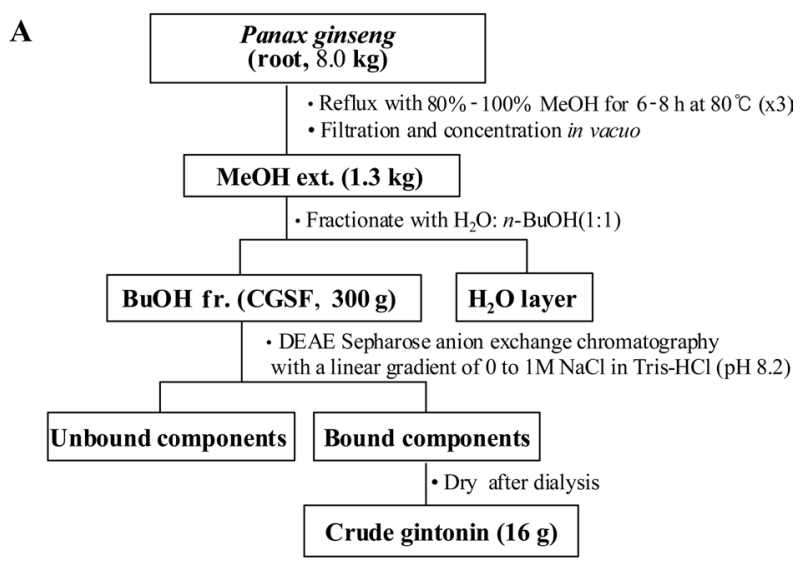

B

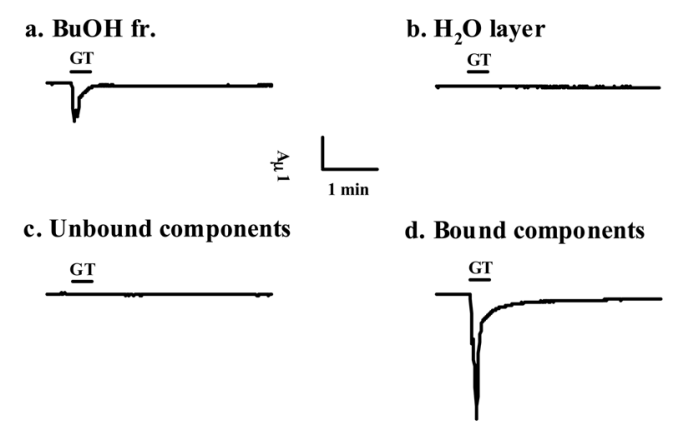

Fig. 1. Diagram for simple preparation of crude gintonin from Panax ginseng root. (A) A complete diagram for crude gintonin preparation from ginseng root. (B) The representative $\mathrm{Ca}^{2+}$-activated $\mathrm{Cl}^{-}$channel $(\mathrm{CaCC})$ current traces are representative of one obtained from each preparation step. Treatment with butanol fraction and the bound component obtained after elution with $\mathrm{NaCl}$ in Tris- $\mathrm{HCl}(\mathrm{pH}$ 8.2) induced endogenous $\mathrm{CaCC}$ activation in Xenopus oocytes, whereas $\mathrm{H}_{2} \mathrm{O}$ layer and unbound component had no effect. The amount of each fraction used to test CaCC activity was $10 \mu \mathrm{g} / \mathrm{mL}$. Inward currents were recorded at $-80 \mathrm{mV}$ holding potential. CGSF, crude ginseng total saponin fraction; fr., fraction; GT, gintonin.

a column packed with DEAE sepharose CL-6B (GE Healthcare) and equilibrated with PBS (pH 7.2). The unbound materials were eluted with the same buffer and the bound materials were eluted with a linear gradient of 0 to $1 \mathrm{M} \mathrm{NaCl}$ in PBS (pH 7.2). The eluted fraction was further dialyzed at $4^{\circ} \mathrm{C}$ for $8 \mathrm{~h}$ with 1,000 -fold excess distilled water (DW) using Spectra/Por dialysis membrane (molecular weight cut off 6,000-8,000; Spectrum Laboratories Inc., Rancho Dominguez, CA, USA) to remove small molecular components such as ginsenosides and other components that might remain in the fraction (16 g) [5]. We labeled this fraction as crude gintonins, which were derived from the root (Fig. 1).

\section{Preparation of gintonin fraction from ginseng stem}

The dried thirty eight grams of 4-year-old red ginseng stems were ground into small pieces $(>3 \mathrm{~mm})$ and re- 
$\mathbf{A}$

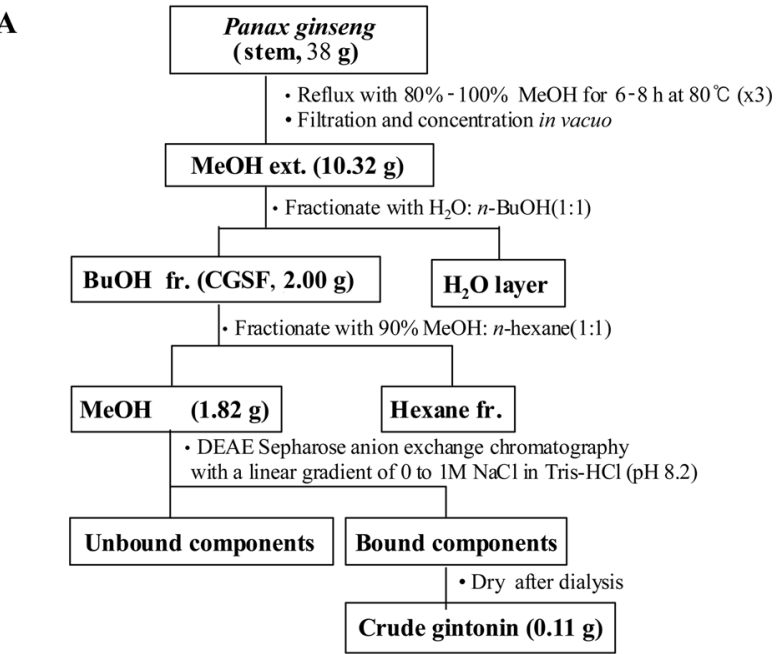

B

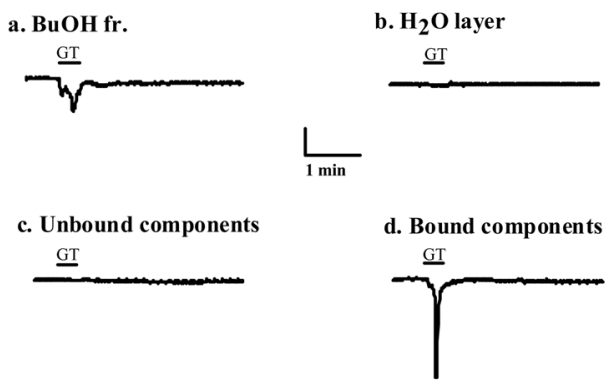

Fig. 2. Diagram for preparation of gintonin fraction from Panax ginseng stem. (A) A complete diagram for crude gintonin preparation from ginseng stem. (B) The representative $\mathrm{Ca}^{2+}$-activated $\mathrm{Cl}^{-}$channel $(\mathrm{CaCC})$ current traces are representative of one obtained from each crude gintonin preparation step. Treatment with butanol fraction and the bound component obtained after elution with $\mathrm{NaCl}$ in Tris- $\mathrm{HCl}$ ( $\mathrm{pH}$ 8.2) induced endogenous CaCC activation in Xenopus oocytes, whereas $\mathrm{H}_{2} \mathrm{O}$ layer and the unbound component had no effect. The amount of each fraction used to test CaCC activity was $10 \mu \mathrm{g} / \mathrm{mL}$. Inward currents were recorded at $-80 \mathrm{mV}$ holding potential. CGSF, crude ginseng total saponin fraction; fr., fraction; GT, gintonin.

fluxed with $80 \% \mathrm{MeOH}$ three times for $8 \mathrm{~h}$ at $80^{\circ} \mathrm{C}$ each. The $\mathrm{MeOH}$ extracts $(10.32 \mathrm{~g})$ concentrated in vacuo were partitioned between $n$ - $\mathrm{BuOH}$ and water. The $n-\mathrm{BuOH}$ fr. $(2 \mathrm{~g})$ of ginseng stems after concentration was further extracted with methanol:hexane (MeOH:Hexane=90:10), while the $\mathrm{MeOH}$ fr. (1.82 g) after concentration was dissolved in PBS (pH 7.2), loaded onto a column packed with DEAE sepharose CL-6B (GE Healthcare) and equilibrated with PBS ( $\mathrm{pH}$ 7.2). The unbound materials were eluted with the same buffer and the bound materials were eluted with a linear gradient of 0 to $1 \mathrm{M} \mathrm{NaCl}$ in PBS ( $\mathrm{pH}$ 7.2). The eluted fraction was further dialyzed at $4^{\circ} \mathrm{C}$ for $8 \mathrm{~h}$ with 1,000 -fold excess DW using Spectra/Por dialysis membrane (molecular weight cut off 6,000-8,000; Spectrum Laboratories Inc.) to remove small molecular components such as ginsenosides and other components that might remain in the fraction $(0.11 \mathrm{~g})$. We labeled this fraction as crude gintonins, which were derived from the stem (Fig. 2).

\section{Preparation of gintonin fraction from ginseng leaf}

The dried $37.3 \mathrm{~g}$ of 4-year-old red ginseng leaves were ground into small pieces $(>3 \mathrm{~mm})$ and refluxed with $80 \% \mathrm{MeOH}$ three times for $8 \mathrm{~h}$ at $80^{\circ} \mathrm{C}$ each. The $\mathrm{MeOH}$ extract $(10.84 \mathrm{~g})$ concentrated in vacuo was partitioned between $n$ - $\mathrm{BuOH}$ and water. $n$ - $\mathrm{BuOH}$ fr. (5.26 g) of ginseng stems after concentration was further extracted with methanol:hexane (MeOH:Hexane=90:10) and methanol fraction $(6.04 \mathrm{~g})$ after concentration was dissolved in PBS (pH 7.2) and was loaded onto a column packed with DEAE sepharose CL-6B (GE Healthcare) and equilibrated with PBS ( $\mathrm{pH}$ 7.2). The unbound materials were eluted with the same buffer and the bound materials were eluted with a linear gradient of 0 to $1 \mathrm{M} \mathrm{NaCl}$ in PBS $\left(\mathrm{pH}\right.$ 7.2). The eluted fraction was further dialyzed at $4^{\circ} \mathrm{C}$ for $8 \mathrm{~h}$ with 1,000-fold excess DW using Spectra/Por dialysis membrane (molecular weight cut off 6,000-8,000; Spectrum Laboratories Inc.) to remove small molecular components such as ginsenosides and other components that might remain in the fraction $(0.3 \mathrm{~g})$. We labeled this fraction as crude gintonins, which were derived from the leaf (Fig. 3).

\section{Gel filtration chromatography}

Gel filtration chromatography of gintonin fractions obtained from ginseng root, stem or leaf was carried out using a Superdex 75 column $(10 \times 300 \mathrm{~mm})$ equilibrated with PBS (pH 7.2) on a BioLogic DuoFlow ${ }^{\mathrm{TM}}$ Chromatography system (Bio-Rad). Fractions were collected with a flow rate of $0.5 \mathrm{~mL} / \mathrm{min}$ and monitored at $280 \mathrm{~nm}$. Each fraction was tested on endogenous $\mathrm{CaCC}$ activations in Xenopus oocytes [5].

\section{Electrophoresis and periodic acid-Schiff base stain- ing}

The crude gintonins from ginseng root, stem, and leaf were subjected to sodium dodecyl sulfate polyacrylamide gel electrophoresis (SDS-PAGE) using 12.0\% separating gel [6]. Crude gintonins (100 $\mu \mathrm{g}$ each) from ginseng root, stem, and leaf were loaded in each lane. After electrophoresis, gintonin bands were visualized by Coomassie Brilliant Blue R-250 staining. For glycoprotein detection, gels were stained using periodic acid-Schiff base staining (PAS). The gel was incubated in $40 \%$ ethanol- $7 \%$ acetic acid in $\mathrm{H}_{2} \mathrm{O}$ for 30 min, followed with $1 \%$ periodic acid- 


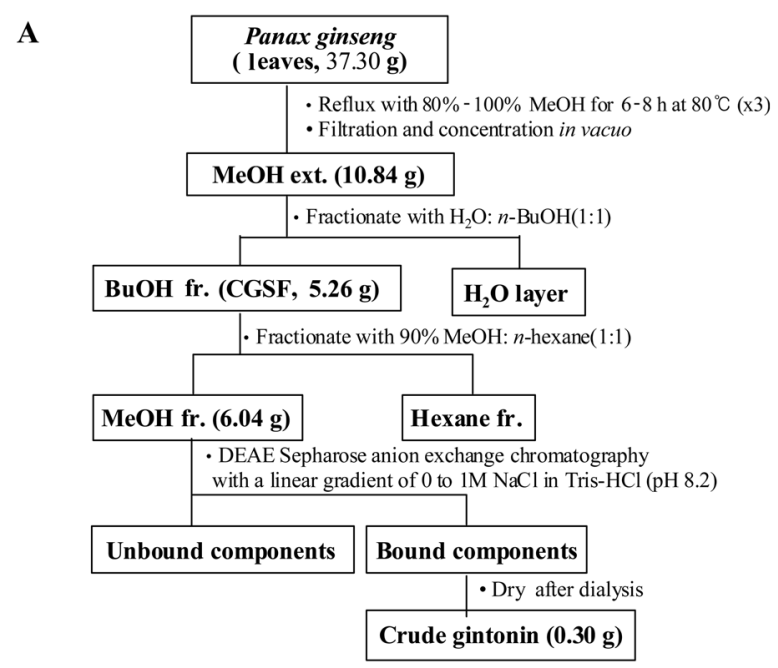

B

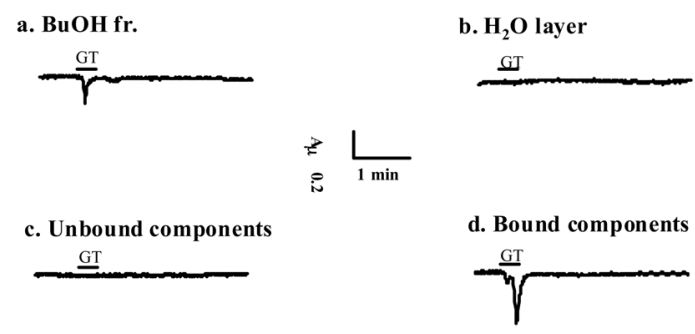

Fig. 3. Diagram for preparation of gintonin fraction from Panax ginseng leaf. (A) A complete diagram for crude gintonin preparation from ginseng leaf. (B) The representative $\mathrm{Ca}^{2+}$-activated $\mathrm{Cl}^{-}$channel $(\mathrm{CaCC})$ current traces are representative of one obtained from each gintonin preparation step. Treatment with butanol fraction and the bound component obtained after elution with $\mathrm{NaCl}$ in Tris- $\mathrm{HCl}(\mathrm{pH}$ 8.2) induced endogenous $\mathrm{CaCC}$ activation in Xenopus oocytes, whereas $\mathrm{H}_{2} \mathrm{O}$ layer and the unbound component had no effect. The amount of each fraction used to test CaCC activity was $10 \mu \mathrm{g} / \mathrm{mL}$. Inward currents were recorded at $-80 \mathrm{mV}$ holding potential. CGSF, crude ginseng total saponin fraction; fr., fraction; GT, gintonin.

$3 \%$ acetic acid for $1 \mathrm{~h}$. The periodic acid solution was removed. Next, Schiff's reagent was added to the gel followed by further incubation for $1 \mathrm{~h}$. Bands of stained glycoproteins developed a pink color and the PAS-stained gels were stored in water after soaking in $7.5 \%$ acetic acid [5].

\section{Gintonin amino acid composition analysis}

Gintonin $(30 \mu \mathrm{g})$ from ginseng root, stem, and leaf was hydrolyzed in vacuo in $6 \mathrm{~N} \mathrm{HCl}$ for $24 \mathrm{~h}$ at $110^{\circ} \mathrm{C}$ for general amino acid analysis. For the analysis of cysteine, gintonin was hydrolyzed in $6 \mathrm{~N} \mathrm{HCl}$ for $24 \mathrm{~h}$ at $110^{\circ} \mathrm{C}$ after peroxidation treatment with formic acid:hydrogen peroxide $=10: 1$. For the analysis of tryptophan, the sample was hydrolyzed in $4 \mathrm{M}$ methanesulfonic acid and 4 $\mathrm{M} \mathrm{KOH}$ was also added. Amino acids converted to phe- nylisothiocyanate derivates were analyzed by high performance liquid chromatography (Hewlett-Packard 1100 series; Hewlett-Packard, Palo Alto, CA, USA) with Waters Nova-Pak C18 column $(3.9 \times 300 \mathrm{~mm})$ at the Korea Basic Science Institute (Seoul, Korea). Protein contents were determined by the Bradford method using BSA as a standard [5].

\section{Carbohydrate composition}

Gintonins from ginseng root, stem, and leaf were hydrolyzed in $2 \mathrm{M}$ trifluoroacetic acid for $4 \mathrm{~h}$ at $100^{\circ} \mathrm{C}$ for neutral sugar and hydrolyzed in $6 \mathrm{~N} \mathrm{HCl}$ for $4 \mathrm{~h}$ at $100^{\circ} \mathrm{C}$ for amino sugar and acid sugar in glass tube. Carbohydrate compositions of gintonin were analyzed by high performance anion exchange chromatography-pulsed ampherometric detection system (HPAEC-PAD system; Dionex, CA, USA) with a CarboPac ${ }^{\mathrm{TM}}$ PA1 column at the Carbohydrate Bioproduct Research Center, Sejong University (Seoul, Korea). The molar ratios of monosaccharides were calculated from peak areas. The carbohydrate contents were also determined by phenol-sulfuric acid method for neutral sugar [7] and anthrone method for acid sugar [8].

\section{Lipid composition analysis}

Gintonins from ginseng root, stem, and leaf were hydrolyzed in $6 \mathrm{~N} \mathrm{HCl}$ for $4 \mathrm{~h}$ at $100^{\circ} \mathrm{C}$ or digested by lipoprotein lipase to confirm lipid and hydrophobic moieties. Acid hydrolyzed or digested gintonins were partitioned between distilled water and $n-\mathrm{BuOH}$. The $n-\mathrm{BuOH}$ layer, after concentration, was further partitioned between distilled water and n-hexane. The $\mathrm{n}$-hexane layer was prepared for lipid and hydrophobic moiety analysis by an Agilent 6890N GC-MS system (Agilent Technologies, Palo Alto, CA, USA) with a DB5-MS capillary column $(30 \mathrm{~cm} \times 250$ $\mu \mathrm{m} \times 0.25 \mu \mathrm{m})$ at the Korea Basic Science Institute and by gas chromatography (Agilent 6890N) equipped with flame ionization detector and a split injection system and fitted with a supelco SPB-1 capillary column $(15 \mathrm{~m} \times 0.32 \mathrm{~mm}$ inside diameter, $0.25 \mathrm{~mm}$ thickness) [5].

\section{Oocyte preparation}

Xenopus laevis frogs were obtained from Xenopus I (Ann Arbor, MI, USA). Their care and handling were in accordance with the highest standards of Konkuk University guidelines. To isolate oocytes, frogs were operated on under anesthesia with an aerated solution of 3-amino benzoic acid ethyl ester. Oocytes were separated by treatment with collagenase and agitation for $2 \mathrm{~h}$ in a $\mathrm{Ca}^{2+}$ free medium containing $82.5 \mathrm{mM} \mathrm{NaCl}, 2 \mathrm{mM} \mathrm{KCl}, 1$ 
$\mathrm{mM} \mathrm{MgCl} 2,5 \mathrm{mM}$ HEPES, $2.5 \mathrm{mM}$ sodium pyruvate, $100 \mathrm{units} / \mathrm{ml}$ penicillin and $100 \mu \mathrm{g} / \mathrm{mL}$ streptomycin. Stage V-VI oocytes were collected and stored in ND96 (96 mM NaCl, $2 \mathrm{mM} \mathrm{KCl}, 1 \mathrm{mM} \mathrm{MgCl}, 1.8 \mathrm{mM} \mathrm{CaCl}_{2}$, and $5 \mathrm{mM}$ HEPES, $\mathrm{pH}$ 7.5) supplemented with $50 \mu \mathrm{g} /$ $\mathrm{mL}$ gentamicin [2]. This oocyte-containing solution was maintained at $18^{\circ} \mathrm{C}$ with continuous gentle shaking and changed daily.

\section{Measurements of endogenous CaCC currents}

Two-electrode voltage-clamp recordings were obtained from individual oocytes placed in a small Plexiglas net chamber $(0.5 \mathrm{~mL})$, which was continuously superfused with the bathing medium (i.e., ND96). The microelectrodes were filled with $3 \mathrm{M} \mathrm{KCl}$ and had a resistance of 0.2-0.7 M $\Omega$. The electrophysiological experiments were performed at room temperature using an oocyte clamp amplifier (OC-725C; Warner Instrument, Hamden, CT, USA). $\mathrm{CaCC}$ was recorded at $-80 \mathrm{mV}$ holding potential, where gintonins were applied to oocytes by bath perfusion [2].

\section{Data analysis}

To obtain the concentration-response curve in the presence of crude gintonin from ginseng root, stem, or leaf the observed peak amplitudes were normalized, plotted and subsequently fitted to the Hill equation below using Origin software (Northampton, MA, USA). $y / y_{\max }=[A]^{\mathrm{n}} /$ $\left([\mathrm{A}]^{\mathrm{n}}+\left[\mathrm{EC}_{50}\right]^{\mathrm{n}}\right)$, where y represents $\%$ activation at given concentration of gintonin, $\mathrm{y}_{\max }$ represents maximal peak current, $\mathrm{EC}_{50}$ is the concentration of gintonin producing half-maximum effect of the control response to gintonin, $\mathrm{A}$ is the concentration of gintonin, and $\mathrm{n}$ is the interaction coefficient. All values were presented as means \pm SEM. The differences between means of control and gintonin treatment data were analyzed using unpaired Student's $t$-test. A value of $p<0.05$ was considered statistically significant.

\section{RESULTS}

\section{A simple procedure for crude gintonin preparation from ginseng root, stem, and leaf}

We found that gintonin is co-fractionated with ginsenosides following butanol extraction, although physicochemical properties of gintonin and ginsenosides differ from each other. Gintonin consists of carbohydrate, lipid, and protein portion [5]. In a previous report, we demonstrated that crude gintonin could be separated from ginsenosides in the butanol fraction through multiple steps using various organic solvents including anion exchange chromatography [5].

The previous method required numerous organic solvents, required a considerable amount of time, and produced a lower yield of gintonin [5]. In the present study, we directly applied the butanol fraction of ginseng root to an anion exchange column since gintonin, but not ginsenosides with anion charges, could bind to the anion column. As shown in Fig. 1B, the unbound components within the column did not induce any activation of $\mathrm{CaCC}$, but the bound portion eluted by $\mathrm{NaCl}$ gradient induced a large inward $\mathrm{Cl}^{-}$current by activating $\mathrm{CaCC}$. These results indicate that the main component for $\mathrm{CaCC}$ activation is gintonin, which carries charges, and that unbound components possibly including ginsenosides and other uncharged or neutral components, had no effects on $\mathrm{CaCC}$ activity. By using a similar method we also prepared crude gintonin from ginseng stem and leaf with additional hexane extraction to further remove hydrophobic components that might exist in stem and leaves (Figs. 2 and 3). We found that the bound components in the anion exchange column also activated $\mathrm{CaCC}$ in ginseng stem and leaf but the degree of $\mathrm{CaCC}$ activation was less than that from the ginseng root. The yield was $0.20,0.29$, and $0.81 \%$ for ginseng root, stem and leaf, respectively. This method produced a higher yield compared to the previous method with less time and less organic solvents [5].

\section{Determination of molecular weight of crude ginto- nin}

We next compared the apparent molecular weight of the gintonin fraction prepared from ginseng root, stem, and leaf, respectively. For this, we performed SDS-PAGE using crude gintonin from ginseng root, stem or leaf. We found a broad but single major band and its apparent molecular weight at approximately $13 \mathrm{kDa}$, revealing the possibility that their molecular weights are nearly identical among all three sources (Fig. 4).

\section{Gintonin fraction from ginseng root, stem, and leaf exhibits different pattern gel filtration chro- matography}

We next compared the patterns of gel filtration chromatography of crude gintonin prepared from ginseng root, stem, and leaf, respectively. As shown in Fig. 5, crude gintonin from ginseng root showed a main peak and minor peaks, whereas crude gintonin prepared from ginseng stem and leaf showed a main peak with additional peaks as shown in ginseng root. The main peaks of ginseng root, stem, and leaf largely exhibited $\mathrm{CaCC}$ activation in Xenopus oocytes (data not shown). Gel 
chromatographic patterns from the gintonin fraction of root, stem, and leaf differ from each other, yet these results indicate that crude gintonin prepared from ginseng root, stem, and leaf has a common main peak that lead to
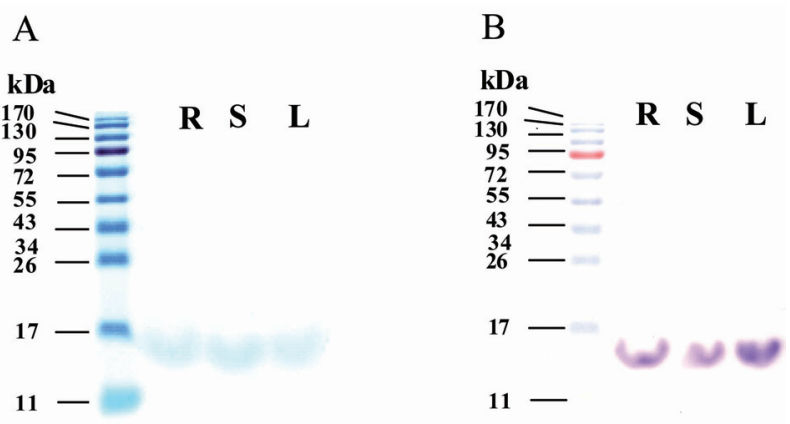

Fig. 4. Sodium dodecyl sulfate polyacrylamide gel electrophoresis (SDS-PAGE) of each gintonin prepared from ginseng root, stem, and leaf. (A) SDS-PAGE of the crude gintonins obtained from anion exchange chromatography. Coomassie Brilliant blue staining was used to stain protein moieties of gintonins. Crude gintonin prepared from ginseng root (R), stem (S), and leaf (L) in SDS-PAGE showed that the apparent molecular weight of gintonin is about $13 \mathrm{kDa}$. (B) Periodic acid-Schiff staining demonstrated that crude gintonins contain carbohydrate moiety.

Table 1. Amino acid composition of gintonins

\begin{tabular}{lrrr}
\hline Amino acid & Root & Stem & Leaf \\
\hline CYA $^{1)}$ & 5.36 & 8.74 & 10.31 \\
ASX $^{2)}$ & 11.26 & 4.98 & 6.71 \\
GLX $^{3)}$ & 7.15 & 5.20 & 5.24 \\
Ser & 5.46 & 4.60 & 5.17 \\
Gly & 10.99 & 15.83 & 18.93 \\
His & 0.00 & 0.00 & 0.00 \\
Arg & 2.57 & 1.32 & 1.38 \\
Thr & 4.46 & 1.76 & 2.25 \\
Ala & 6.71 & 3.87 & 3.86 \\
Pro & 6.48 & 5.14 & 5.24 \\
Tyr & 1.31 & 0.99 & 0.84 \\
Val & 5.06 & 4.70 & 3.49 \\
Met & 0.89 & 1.19 & 0.70 \\
Ile & 5.39 & 8.15 & 6.78 \\
Leu & 7.98 & 5.39 & 5.34 \\
Phe & 11.45 & 17.42 & 14.89 \\
Trp & 1.10 & 1.86 & 1.65 \\
Lys & 6.38 & 8.85 & 7.22 \\
\hline
\end{tabular}

The detail methods for amino acid compositions of each gintonin are described in Materials and Methods section.

Data are presented as percentage.

${ }^{1)}$ The sum of cysteine and cystine.

${ }^{2)}$ The sum of asparagine and aspartic acid.

${ }^{3)}$ The sum of glutamine and glutamic acid.
CaCC activation.

\section{Amino acid, carbohydrates, and lipid composition of gintonin prepared from ginseng root, stem, and leaf}

The contents of total proteins in crude gintonin analyzed by Bradford method were approximately $25 \%$, $13.8 \%$, and $12.5 \%$ for ginseng root, stem, and leaf, respectively. Thus, protein contents of crude gintonin prepared from root were about three times higher than that from stem and leaf, leading to the possibility that stem and leaf contain other unidentified components besides protein. Amino acid composition of individual gintonins was summarized in Table 1.

As shown in Fig. 4 (right panel), the bands representative of crude gintonin from SDS-PAGE are single but broad and are not strongly stained with Coomassie Brilliant blue staining, indicating the possibility that crude gintonin might contain carbohydrate moieties. Therefore, we used PAS technique to determine whether gintonin contains carbohydrates as a previous report had suggested [5]. Crude gintonin from ginseng root, stem, and leaf was strongly stained by PAS as shown in Fig. 4B. Next, we examined carbohydrate composition of gintonins using the HPAEC-PAD system. Table 2 showed that carbohydrate compositions of gintonin prepared from ginseng root, stem, and leaf slightly differ from each other. Glucose was a major component (Fig. 6). Finally, the contents of total carbohydrate in crude gintonin were about $21.9 \%, 16.5 \%$, and $33 \%$ for ginseng root, stem, and leaf, respectively. These results confirmed once again

Table 2. Carbohydrate composition of gintonins

\begin{tabular}{lrrr}
\hline Carbohydrates & Root & Stem & Leaf \\
\hline Galactosamine & 3.59 & 1.66 & 1.89 \\
Galactose & 19.72 & 12.13 & 53.09 \\
Glucose & 70.74 & 79.43 & 45.02 \\
Glucuronic acid & 5.95 & 6.78 & - \\
\hline
\end{tabular}

The detail methods for carbohydrate compositions of each gintonin are described in Materials and Methods section.

Data are presented as percentage.

Table 3. Lipids composition of gintonins

\begin{tabular}{lrrr}
\hline \multicolumn{1}{c}{ Lipids } & Root & Stem & Leaf \\
\hline Palmitic acid (16:0) & 38.79 & 35.78 & 40.33 \\
Stearic acid (18:0) & 2.29 & 2.08 & 2.33 \\
Linoleic acid (18:2) & 52.66 & 57.34 & 57.34 \\
Others & 6.26 & 4.81 & - \\
\hline
\end{tabular}

The detail methods for lipid compositions of each gintonin are described in Materials and Methods section.

Data are presented as percentage. 
A. Root

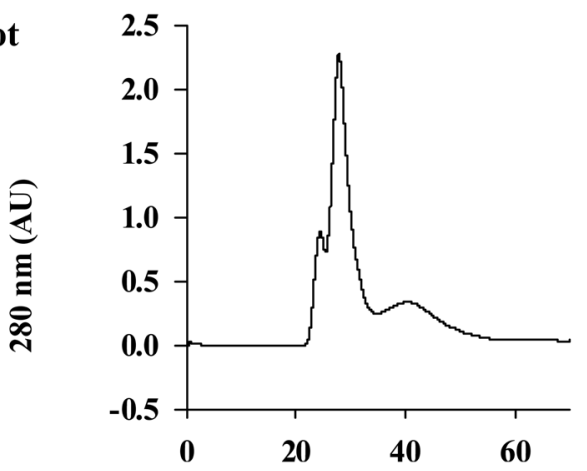

B. Stem

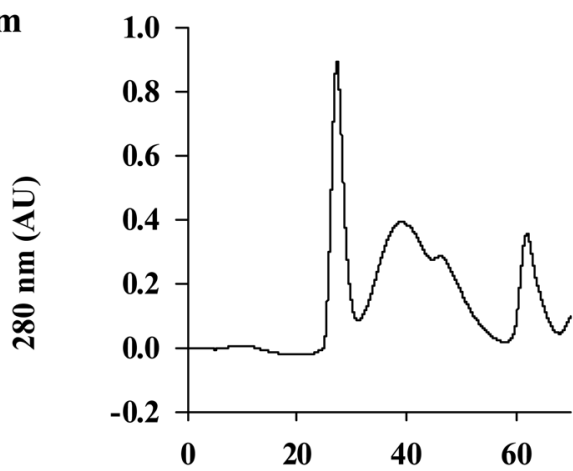

C. Leaf

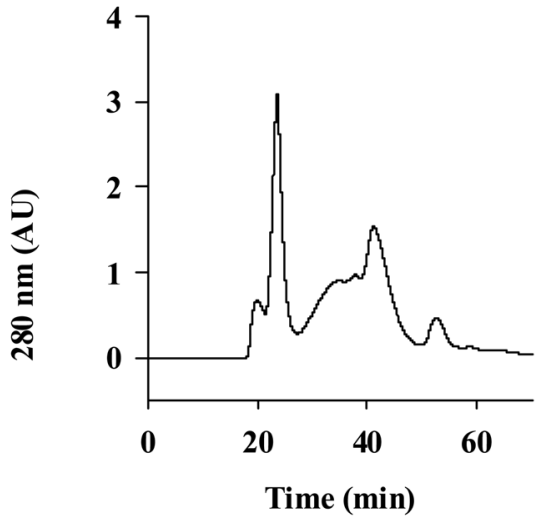

Fig. 5. Elution patterns of gintonin prepared from ginseng root, stem, or leaf by gel chromatography. Gel filtration chromatograms on Superdex 75 column with phosphate buffer saline $(\mathrm{pH} 7.2)$ of crude gintonin prepared from ginseng root (A), stem (B) or leaf (C). Analysis through gel filtration chromatograms showed that the main peaks of crude gintonin prepared from root, stem, or leaf are almost same but ginseng stem and leaf showed an additional peak after the main peak. We found that the main peak was active for the activation of $\mathrm{Ca} 2+$-activated $\mathrm{Cl}$ - channel.

that gintonins are glycoproteins.

We also examined lipid moieties. As shown in Table 3, crude gintonin of ginseng root, stem, and leaf contained fatty acids such as palmitic, stearic, linoleic acid, and other minor fatty acids. The portion of polyunsaturated fatty acid, linoleic acid in crude gintonin of ginseng
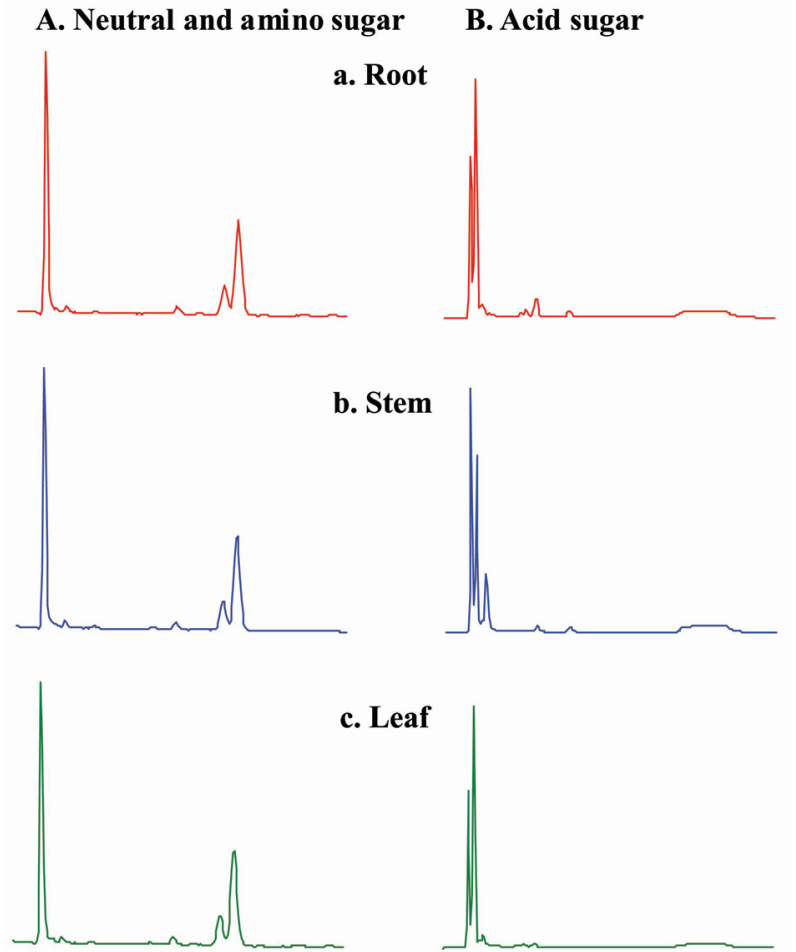

c. Leaf
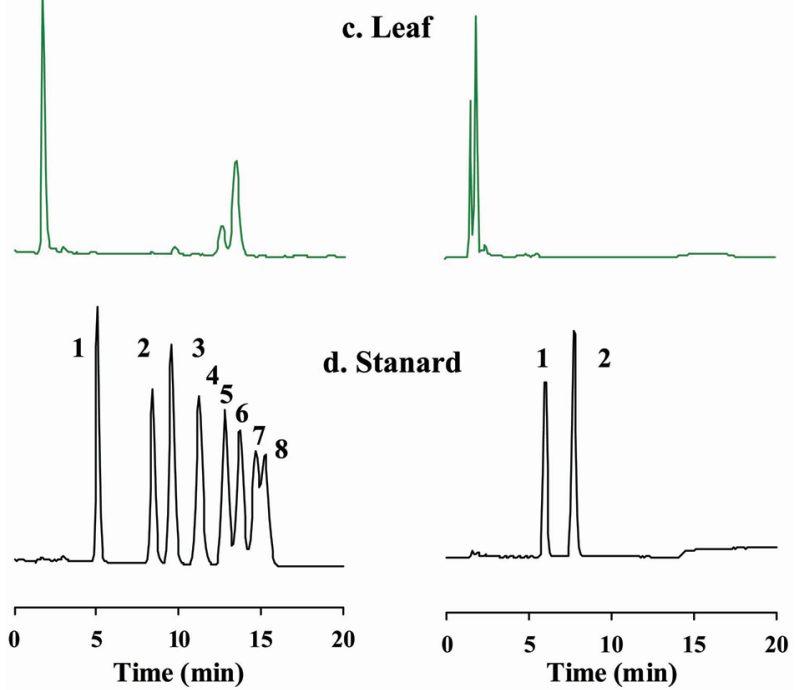

Fig. 6. Analysis of carbohydrate components of crude gintonin prepared from ginseng root, stem, or leaf by HPAEC-PAD chromatograms. (A-C) HPAEC-PAD chromatograms show that gintonins are composed of two different kinds of neutral sugars:glucose and galactose, one amino sugar, galactosamine, and one acid sugar, glucuronic acid. (A) Std stands for standard carbohydrates used; 1. Lfucose, 2. L-rhamnose, 3. D-galactosamine, 4. D-glucosamine, 5. Dgalactose, 6. D-glucose, 7. D-mannose, 8, D-xylose. (B) Acid sugar; 1. Galacturonic acid, 2. Glucuronic acid.

root, stem, and leaf were greater than saturated fatty acids such as palmitic and stearic acid (Fig. 7). We found linoleic acid was the predominant lipid in crude gintonin of ginseng root, stem, and leaf. Next, we estimated total contents of lipid moiety in gintonins by gas chromatography. The contents of total lipids in crude gintonin were $25 \%, 13 \%$, and $16 \%$ for ginseng root, stem, and leaf, respectively, once again reiterating that crude gintonin prepared from ginseng stem and leaf is composed of glycolipoproteins. 


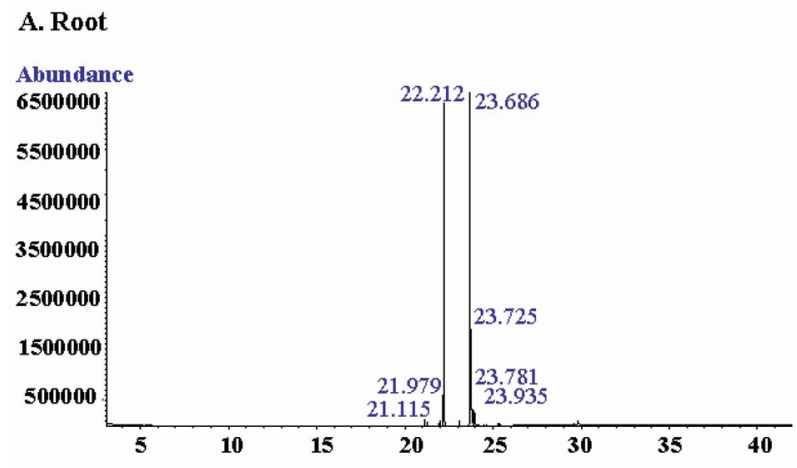

B. Stem

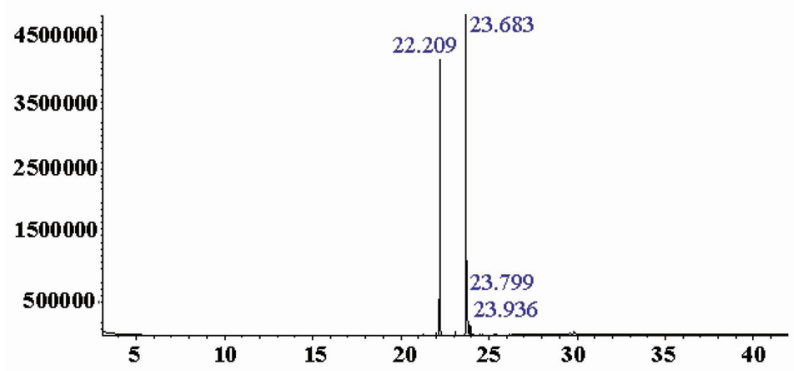

C. Leaf

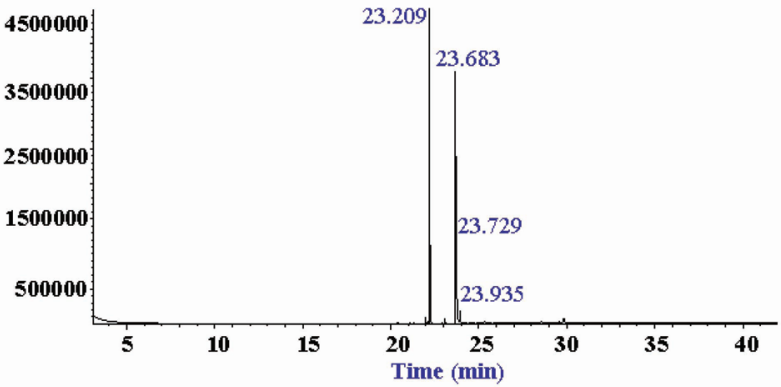

Fig. 7. GC-MS spectral analysis of lipid components of crude gintonin prepared from ginseng root, stem, or leaf. Acid hydrolyzed gintonin was partitioned between distilled water and n-butanol $(n-\mathrm{BuOH})$. The $n-\mathrm{BuOH}$ layer, after concentration, was further partitioned between distilled water and n-hexane. The $n$-hexane layer was subjected to GC-MS with a DB5-MS capillary column. Several peaks were present in the hexane fraction of gintonin and were identified as palmitic acid, stearic acid, oleic acid, linoleic acid ester or free form. The 22.11 peak of palmitic acid $\left(\mathrm{C}_{16: 0}\right)$ and 23.59 peak of linoleic acid $\left(\mathrm{C}_{18: 2}\right)$ were dominant fatty acid in gintonins.

\section{Effects of gintonin prepared from ginseng root, stem, and leaf on endogenous CaCC in Xenopus oocytes}

We compared the degree of activation of $\mathrm{CaCC}$ of crude gintonin prepared from ginseng root, stem, and leaf. As shown in Fig. 8, treatment of crude gintonin prepared from ginseng root, stem, and leaf induced large inward $\mathrm{Cl}^{-}$currents in a concentration-dependent manner

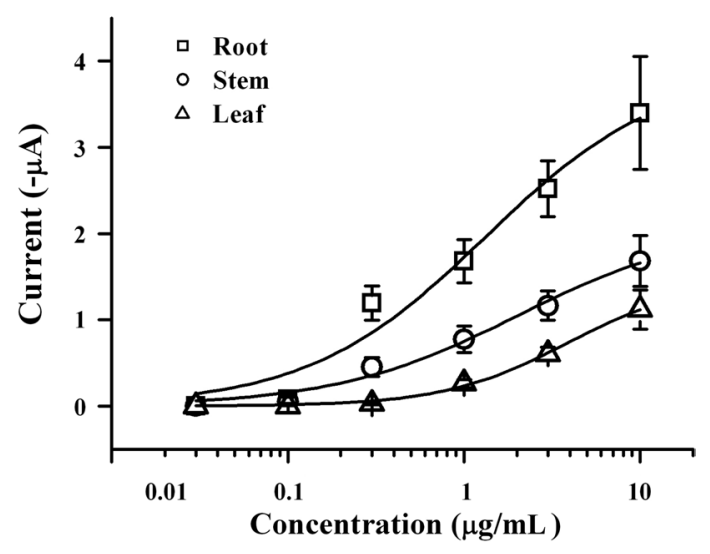

Fig. 8. Crude gintonin prepared from ginseng root, stem, or leaf activates $\mathrm{Ca}^{2+}$-activated $\mathrm{Cl}^{-}$channel $(\mathrm{CaCC})$ with concentration-dependent manner. Crude gintonin prepared from ginseng root, stem, and leaf increased inward $\mathrm{CaCC}$ currents in a concentration-dependent manner. The order of gintonin potency for $\mathrm{CaCC}$ activation was root $>$ stem $>$ leaf. Data represent means \pm SEM $(n=7-8)$.

at $-70 \mathrm{mV}$ holding potential (Fig. 8). However, there was a difference among them for the potency for $\mathrm{CaCC}$ activation. The order of potency for CaCC activation by crude gintonin was root $>$ stem $>$ leaf. The $\mathrm{ED}_{50}$ was $1.4 \pm 1.4$, 4.5 \pm 5.9 , and $3.9 \pm 1.1 \mu \mathrm{g} / \mathrm{mL}$ for ginseng root, stem, and leaf, respectively. Thus, these results indicate that although crude gintonin prepared from ginseng root, stem, and leaf are similar to each other in terms of molecular weights and physico-chemical properties, there are differences in the degree of activating $\mathrm{CaCC}$ in Xenopus oocytes.

In the previous report, we demonstrated that ginseng root contains novel glycolipoprotein ingredients [5]. We named, which we named it as gintonin. Although gintonin was co-fractionated with ginsenosides in the butanol fraction, gintonin showed several distinguishing characteristics from ginsenosides. For example, gintonin carries charges, since gintonin consists of carbohydrates, lipids, and proteins, whereas in general we have determined that ginsenosides are one of neutral glycosides without any charges. The apparent molecular weight of gintonin is about $13 \mathrm{kDa}$ but that of ginsenosides ginsenosides overall is about $0.8-1.3 \mathrm{kDa}$. More importantly, gintonin treatment caused activation of $\mathrm{Ca}^{2+}$-dependent ion channels such as the endogenous $\mathrm{CaCC}$ in Xenopus oocytes through mobilization of $\left[\mathrm{Ca}^{2+}\right]_{i}$, but ginsenosides did not [5].

\section{DISCUSSION}

In the present study, we report two major findings. First, we confirmed that ginseng stem and leaf con- 
tain gintonin, in addition to the root that was originally known. Second, we have developed a simple method for gintonin preparation from ginseng root, stem, and leaf. The simple method for crude gintonin preparation from ginseng root, stem, and leaf has at least three advantages as compared to previous method. First, the newly developed method requires a significantly lesser amount of organic solvents than the previous one. Second, the newly developed method takes less time for crude gintonin preparation, and finally third, the gintonin yield using this method is much higher than the previous method and we can apply this procedure to prepare a massive amount of gintonin using ginseng stem and leaf, which are otherwise usually discarded.

We observed some similarities and differences among gintonin prepared from ginseng root, stem, and leaf, respectively. Their molecular weights in SDS-PAGE were nearly identical (Fig. 4). In amino acid compositions, crude gintonin prepared from ginseng root, stem, and leaf contained high amounts of hydrophobic amino acids including phenyalanine, leucine, isoleucine, alanin and proline. Interestingly, phenylalanine was the most abundant amino acid in all gintonin fractions prepared from root, stem and leaf. Next, glycine was also abundant in all crude gintonin (Table 1). In carbohydrate compositions, glucose was the most abundant component in gintonin prepared from ginseng root, stem, and leaf (Table 2). Linoleic acid (C18:2) was the major lipid component in gintonin overall and was followed by palmitic acid (C16:0). Stearic acid (C18:0) was only a minor component (Table 3). Thus, crude gintonins of ginseng root, stem, and leaf are glycolipoproteins. Currently, we do not know how carbohydrate, lipid and protein components combine with each other to form the gintonin structure, and their respective roles in $\mathrm{CaCC}$ activation. In addition, we could not exclude the possibility that gintonin content or composition in ginseng root, stem, and leaf might vary from batch-to-batch and from different sources of ginseng. Further studies will be needed to establish standardization of gintonin and will be required to elucidate the physico-chemical properties of gintonin and the role(s) of each portion of ginseng.

In our previous study we demonstrated that gintonin from ginseng root induced $\mathrm{CaCC}$ activation in Xenopus oocytes through mobilization of $\left[\mathrm{Ca}^{2+}\right]_{i}[5]$. Interestingly, when we compared the potency for endogenous $\mathrm{CaCC}$ activation in Xenopus oocytes using gintonin fraction prepared from ginseng root, stem, and leaf, we found that crude gintonin prepared from ginseng root was much more potent than that from stem and leaf for the activation of $\mathrm{CaCC}$. Thus, the potency of gintonin from ginseng stem for the activation of $\mathrm{CaCC}$ was half that of gintonin from ginseng root. The potency of gintonin from ginseng leaf was about one-third of gintonin prepared from ginseng root (Fig. 8). We could not clearly explain why there are some differences for the activation potency of $\mathrm{CaCC}$ among the different sources of gintonin. A possible reason is that gintonin prepared from ginseng stem and leaf might contain other unidentified components that were not revealed in ginseng root by gel chromatography (Fig. 5), although we have extracted with hexane to remove hydrophobic components that may have remained in the butanol fraction.

Usually, ginseng stem and leaf are discarded as waste after ginseng root harvest, since the traditional herbal medicines only use ginseng root. Although the amount of discarded ginseng stem and leaf annually are considerably large compared to the amount of ginseng root used, no efficient methods were available to utilize ginseng stem and leaf until now. In the present study, we found that ginseng stem and leaf also contain gintonin and could be prepared from a simple preparation method similar to ginseng root. Therefore, we have at least two benefits for gintonin preparation from ginseng stem and leaf: we can utilize ginseng stem and leaf that are usually discarded after ginseng is harvested as described above for crude gintonin preparation instead of ginseng root, which is quite expensive to buy. Additionally, we could obtain a large amount of gintonin from ginseng stem and leaf, if the methods for mass processing of ginseng stem and leaf are developed.

Taken together, we identified that ginseng stem and leaf in addition to ginseng root contain a novel glycolipoprotein, which we refer to as gintonin, that causes the activation of CaCCs in Xenopus oocytes through mobilization of $\left[\mathrm{Ca}^{2+}\right]_{\mathrm{i}}$. Since elevation of intracellular $\mathrm{Ca}^{2+}$ as a second messenger plays an important role in a variety of cellular functions [9], the present findings also demonstrate the possibility that gintonins prepared from ginseng stem and leaf as well as ginseng root could be utilized for the explanation of $\mathrm{Ca}^{2+}$-mediated physiological and pharmacological effects of ginseng.

\section{ACKNOWLEDGEMENTS}

This work was supported by Basic Science Research Program through the National Research Foundation of Korea (NRF) funded by the Ministry of Education, Science and Technology (R01-2008-000-10448-0), Priority Research Centers Program through the NRF funded 
by the Ministry of Education, Science and Technology (2009-0093824), and BK21 to Seung-Yeol Nah.

\section{REFERENCES}

1. Nah SY. Ginseng: recent advances and trends. Korean J Ginseng Sci 1997;21:1-12.

2. Choi S, Kim HJ, Ko YS, Jeong SW, Kim YI, Simonds WF, Oh JW, Nah SY. G alpha(q/11) coupled to mammalian phospholipase $\mathrm{C}$ beta 3-like enzyme mediates the ginsenoside effect on $\mathrm{Ca}(2+)$-activated $\mathrm{Cl}(-)$ current in the Xenopus oocyte. J Biol Chem 2001;276:48797-48802.

3. Lee JH, Jeong SM, Lee BH, Noh HS, Kim BK, Kim JI, Rhim H, Kim HC, Kim KM, Nah SY. Prevention of ginsenoside-induced desensitization of $\mathrm{Ca}^{2+}$-activated $\mathrm{Cl}^{-}$ current by microinjection of inositol hexakisphosphate in Xenopus laevis oocytes: involvement of GRK2 and betaarrestin I. J Biol Chem 2004;279:9912-9921.
4. Jeong SM, Lee JH, Kim S, Rhim H, Lee BH, Kim JH, Oh JW, Lee SM, Nah SY. Ginseng saponins induce store-operated calcium entry in Xenopus oocytes. Br J Pharmacol 2004;142:585-593.

5. Pyo MK, Choi SH, Hwang SH, Shin TJ, Lee BH, Lee SM, Lim YH, Kim DH, Nah SY. Novel glycolipoproteins from ginseng. J Ginseng Res 2011;35:92-103.

6. Bradford MM. A rapid and sensitive method for the quantitation of microgram quantities of protein utilizing the principle of protein-dye binding. Anal Biochem 1976;72:248-254.

7. Kobata A. Structures and functions of the sugar chains of glycoproteins. Eur J Biochem 1992;209:483-501.

8. Scott TA Jr, Melvin EH. Determination of dextran with anthrone. Anal Chem 1953;25:1656-1661.

9. Berridge MJ, Bootman MD, Roderick HL. Calcium signalling: dynamics, homeostasis and remodelling. Nat Rev Mol Cell Biol 2003;4:517-529. 